\title{
Développement chez des Moustiques de trois Filaires de Lézards sud-américains du genre Oswaldofilaria*
}

\author{
par O. BAIN et A.-G. CHABAUD \\ Laboratoire de Zoologie (Vers), associé au C.N.R.S. \\ Muséum national d'Histoire naturelle, 43, ruc Cuvier, F 75231 Paris Cedex 05.
}

Résumé.

Le développement d'O. petersi, $O$. belemensis et $O$. spinosa se déroule comme celui d'O. bacillaris: les larves sont situées dans le tissu adipeux de divers moustiques; les stades infestants ont une cuticule ornée d'arêtes longitudinales, une queue longue munie de deux languettes subterminales et un œsophage glandulaire bien développé; l'ébauche génitale $q$ est située dans la moitié antérieure du corps, mais reste proche de la région médiane. Ce caractère oppose ces espèces aux autres Filaires vivipares et souligne l'originalité des Oswaldofilariinae.

\section{Summary.}

Development in Mosqui:oes of three Oswaldofilaria from South-american lizards.

The development of $O$. petersi, $O$. belemensis and $O$. spinosa is similar to that of $O$. bacillaris: the larvae are in the adipose tissue of various mosquitoes; the infective stages are characterized by the longitudinal salient ridges of the cuticule, the long tail ended by two lappets, the well-developed glandular oesophagus; the $q$ genital anlage lies in the anterior half part of the body, but is not very far from the median line. This character opposes these species to the other viviparous Filariae and stresses the originality of the Oswaldofilariinae.

${ }^{*}$ *) Ce travail a pu être effectué grâce à une subvention de l'Organisation mondiale de la Santé. Nous remercions vivement le $\mathrm{D}^{r}$ Lainson et ses collaborateurs, ainsi que le $\mathrm{P}^{\mathrm{r}}$ Peters, pour l'aide qu'ils nous ont apportée.

Annales de Parasitologie humaine et comparée (Paris), t. 50, $\mathrm{n}^{\circ} 2$ 


\section{Introduction}

$\mathrm{Au}$ cours de plusieurs missions au Centre Evandro Chagas (Wellcome Unit) à Belem, Brésil, de nombreux lézards parasités par des Oswaldofilariinae (Piratuba Lent et Freitas, 1941 ; Piratuboides Bain et Sulahian, 1975 ; Oswaldofilaria Travassos, 1933) ont été récoltés. Divers Moustiques ont été gorgés sur ces lézards : Culex fatigans, Culex pipiens et, plus rarement, Anopheles stephensi et Aedes aegypti. Les trois espèces qui se sont développées jusqu'au stade infestant, et qui sont l'objet de cette note, appartiennent au genre Oswaldofilaria; ce sont: O. petersi Bain et Sulahian, 1975, parasite de Tupinambis nigropunctatus Spix, O. belemensis Bain et Sulahian, 1975, parasite de Dracaena guyanensis Daudun, et $O$. spinosa Bain et Sulahian, 1975, parasite de Mabuya mabouia mabouia Lacépède.

\section{Oswaldofilaria petersi}

\section{Allure du développement.}

Deux vecteurs ont été utilisés avec succès au laboratoire: Culex fatigans (élevage de l'Institut Chagas, Belem) et Culex pipiens (souche autogène du Laboratoire d'Ecologie parasitaire, Montpellier). A $25^{\circ}$ le développement s'effectue en 16 jours, dans le tissu adipeux, abdominal et thoracique et les larves infestantes se répartissent dans la trompe, la tête et le reste du corps.

\section{Croissance et dimensions.}

La larve, dès le début courte et trapue, ne se raccourcit pas; à la mue 1, elle a largement doublé de longueur et s'est considérablement épaissie; pendant le stade II la croissance en longueur est accélérée ; à la mue 2, l'exuvie est refoulée avec le bouchon cuticulaire, en même temps que l'intestin et le rectum communiquent et que les déchets accumulés dans l'intestin pendant le développement larvaire sont expulsés; le stade infestant est mince et très mobile et ses structures sont moins nettes que chez les stades précédents.

Microfilaire (fig. $1 \mathrm{~A}$ ): les microfilaires sanguines en coloration vitale sont longues de 66 à $72 \mu$ et larges de $8 \mu$ dans la moitié antérieure du corps qui est la région la plus épaisse ; seul le corps interne est visible, à 43-45 $\mu$ de l'extrémité céphalique.

Stade $I$ (fig. 1 et $2 A, B, C$ ).

Larve de $48 \mathrm{~h}$ : (fig. $1 \mathrm{~B}$ ) : corps long de $87 \mu$ et large de $9 \mu$ (dans la moitié postérieure du corps, qui est maintenant la plus large); anneau nerveux, noyau 


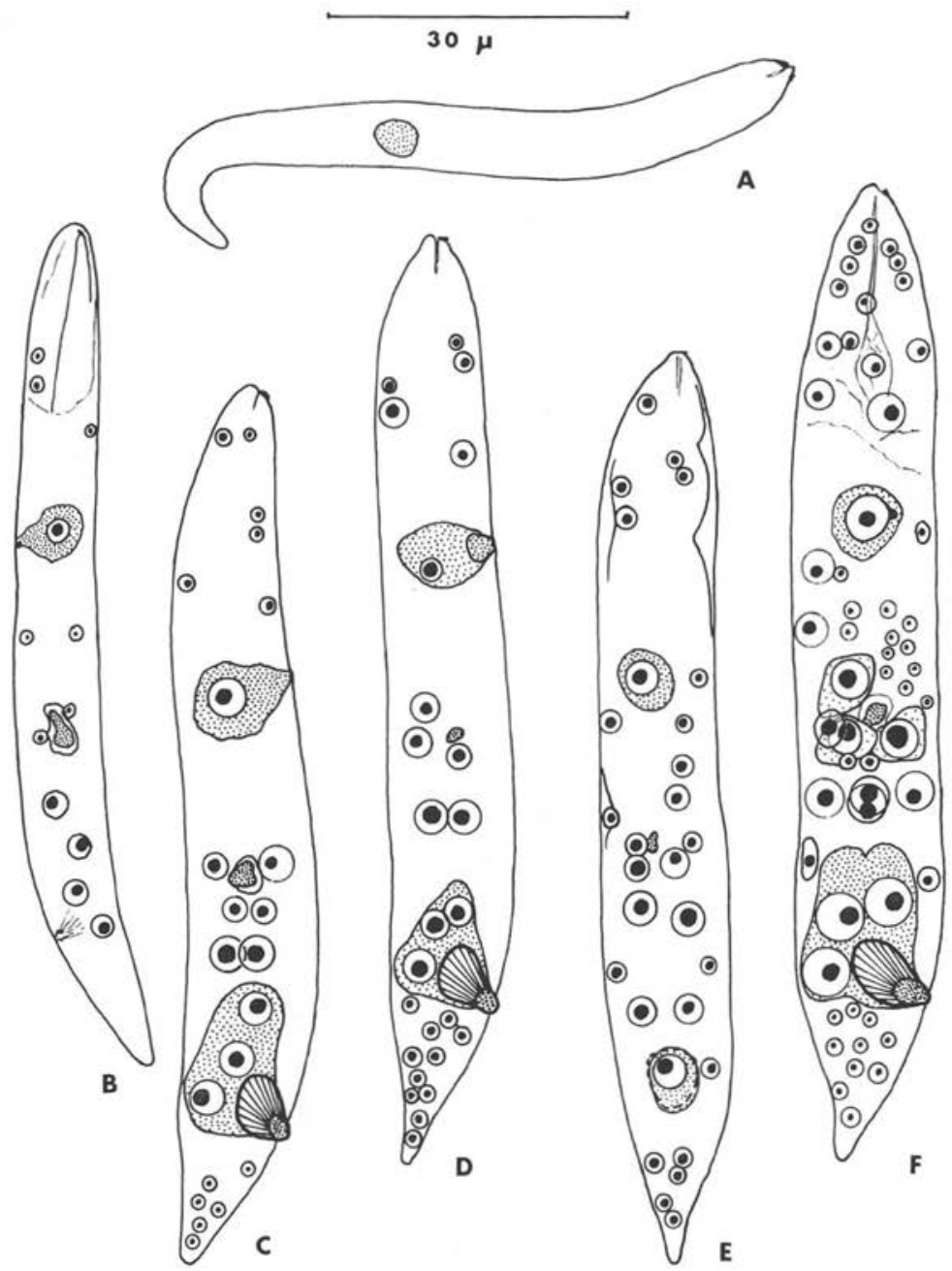

FIG. 1. - Oswaldofilaria petersi. A : microfilaire en coloration vitale; B: larve de 48 h; $\mathrm{C}, \mathrm{D}$ et $\mathrm{E}$ : larve de $72 \mathrm{~h}$ en vues latérale et dorsale; F : larve de $92 \mathrm{~h}$

excréteur, corps interne et noyau $\mathrm{R}_{1}$, respectivement à $20 \mu, 32 \mu, 52 \mu$ et $57 \mu$ de l'extrémité antérieure; queue longue de $15 \mu$.

Larve de $72 \mathrm{~h}$ (fig. $1 \mathrm{D}$ ) : corps long de $87 \mu$ et large de $13 \mu ; \mathrm{R}_{1} \times 2$ à $55 \mu$ de l'apex; rectum haut de $15 \mu$, queue longue de $16 \mu$.

Larve de 5 jours (fig. $2 \mathrm{~A}$ ) : corps long de $178 \mu$ et large de $18 \mu$; pore excréteur à $38 \mu$ de l'apex; ébauches œsophagienne, intestinale et rectale longues respectivement de $65 \mu, 20 \mu$ et $25 \mu$; queue longue de $25 \mu$. 

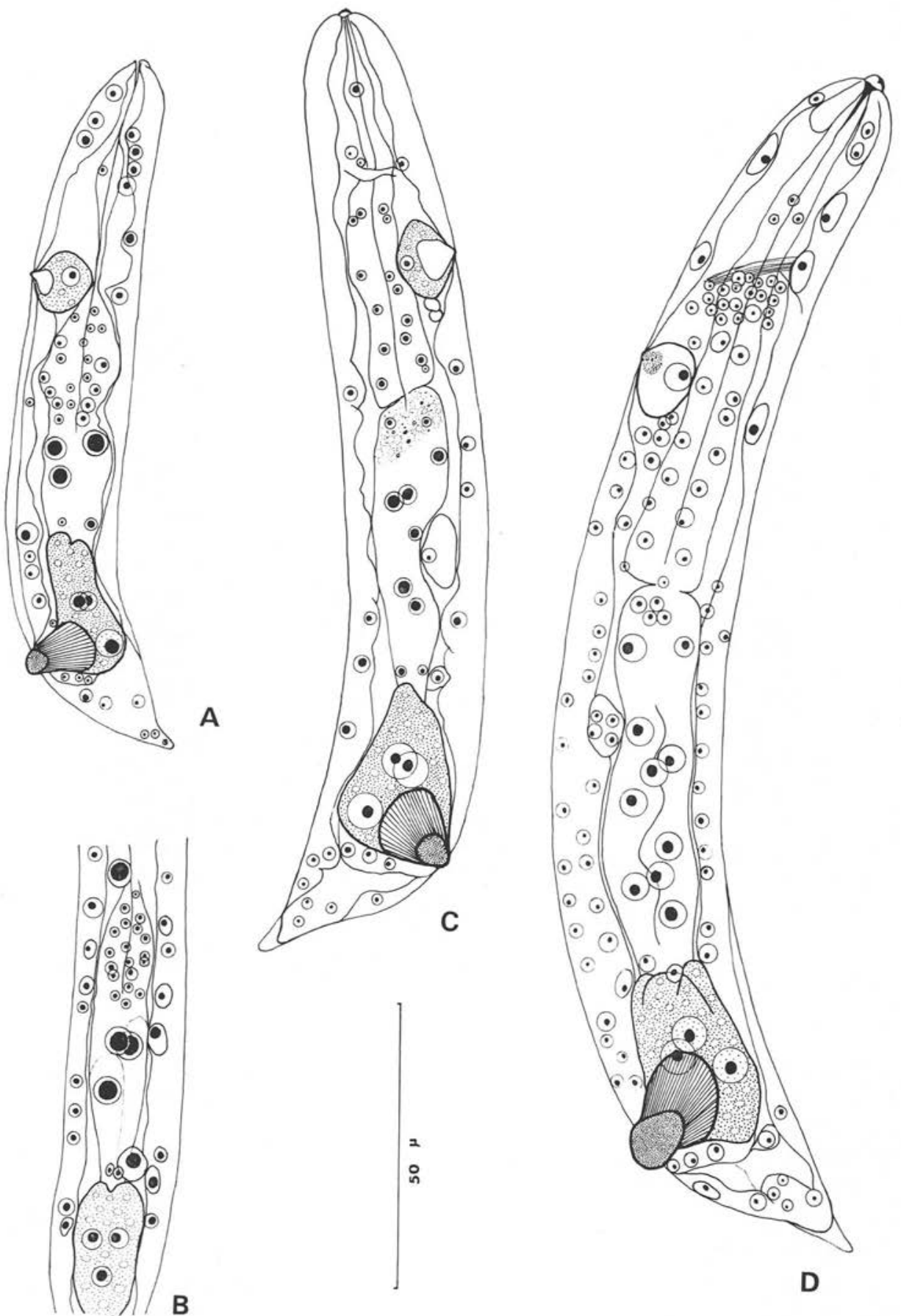

FIG. 2. - Oswaldofilaria petersi. A : larve de 5 jours; B : idem; vue dorsale; $\mathrm{C}: 7^{\circ}$ jour, début de la mue $1 ; \mathrm{D}$ : très jeune stade II. 
Mue 1, début du décollement au $7^{\circ}$ jour (fig. 2 C): corps long de $177 \mu$ et large de $27 \mu$; anneau nerveux et pore excréteur respectivement à $29 \mu$ et $43 \mu$ de l'extrémité antérieure; œsophage, intestin et rectum longs rspectivement de $67 \mu$, $50 \mu$ et $32 \mu$; ébauche génitale $30 \mu$ en arrière de la jonction œsophage-intestin ; queue longue de $30 \mu$.

Stade II, larve de 13 jours (fig. 4 B) : corps long de $445 \mu$ et large de $29 \mu$; capsule buccale haute de $7 \mu$, anneau nerveux et pore excréteur respectivement à $60 \mu$ et $72 \mu$ de l'extrémité antérieure; œsophage, intestin, rectum et queue longs respectivement de $140 \mu, 235 \mu, 38 \mu$ et $25 \mu$.

Mue 2, exuviation le $16^{\mathrm{e}}$ jour (fig. 4C) : corps long de $700 \mu$ et large de $20 \mu$; anneau nerveux à $70 \mu$ de l'apex; œsophages musculaire et glandulaire longs respectivement de $78 \mu$ et $230 \mu$; rectum haut de $35 \mu$; queue longue de $38 \mu$ (autre spécimen: corps long de $940 \mu$ ).

Stade infestant: corps long de 915 à $1180 \mu$ et large de 19 à $20 \mu$; pour une larve $q$ de $915 \mu$, capsule buccale haute de $10 \mu$; anneau nerveux et pore excréteur respectivement à 84 et $124 \mu$ de l'extrémité antérieure ; œsophages musculaire et glandulaire longs de 118 et $337 \mu$; ébauche génitale à $465 \mu$ de la tête ; rectum et queue longs de $22 \mu$ et $46 \mu$.

\section{Etude morphologique.}

Le développement larvaire se déroule dans son ensemble comme chez $O$. bacillaris ( $C f$. Prod'hon et Bain, 1972) et peut être suivi sur les figures $l$ à 5, auxquelles sont ajoutées quelques précisions qui nous paraissent utiles.

- Microfilaire (fig. $1 \mathrm{~A}$ ) : en coloration vitale, les cellules excrétrices et $\mathrm{R}_{1}$ à $\mathrm{R}_{4}$ ne sont pas discernables, tant les noyaux somatiques sont tassés; la tête est nettement pointue, ornée de 2 formations cuticulaires subapicales; le corps interne est sphérique.

- Organogénèse du $1^{\text {er }}$ stade (fig. $1 B$ à $F$ et fig. $2 A$ à $C$ ) :

Les deux premières divisions de $R_{1}$ s'effectuent le $3^{e}$ et le $4^{e}$ jour. En avant des 4 cellules filles de $R_{1}$, les cellules intestinales, au nombre de 3 ou 4 , s'organisent autour du corps interne (fig. $1 B$ à $F$ ); celui-ci disparaît vers le $5^{\circ}$ jour, au moment où le cordon digestif est déjà bien ébauché (fig. $2 \mathrm{~A}$ ). A la mue 1 , les cellules œsophagiennes et intestinales commencent à se multiplier (fig. 2 C).

- Forme infestante (fig. $4 \mathrm{D}$ à $F$ et fig. 5): la bouche et la capsule buccale sont aplaties latéralement. $\mathrm{La}$ cuticule du corps forme des arêtes longitudinales (fig. $5 A$ et $C$ ). L'œsophage est bien divisé avec une portion glandulaire environ deux fois plus longue que la portion musculaire. Le rectum est allongé, la gueue est 


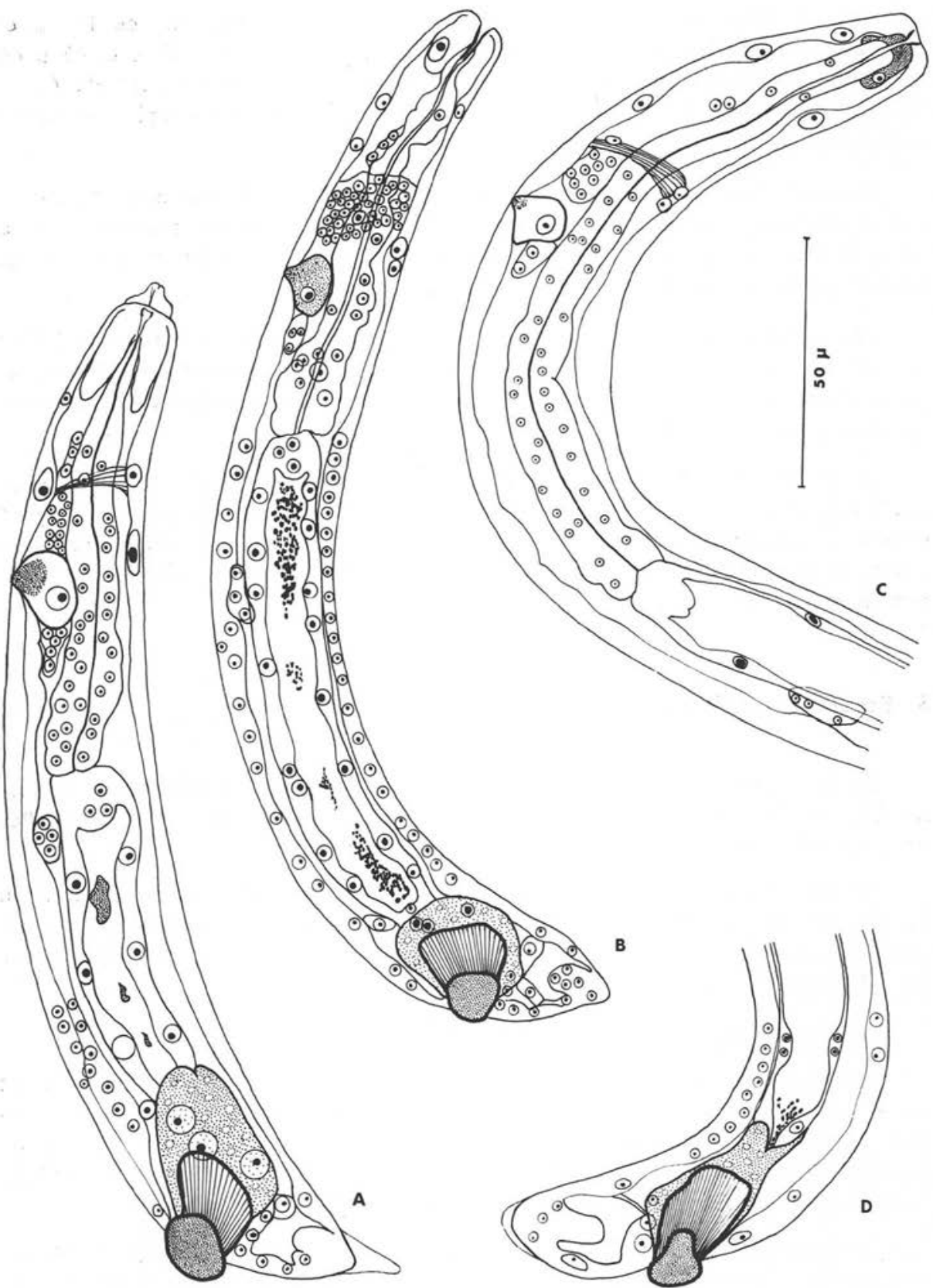

Fig. 3. - Oswaldofilaria petersi; $2^{\mathrm{e}}$ stade larvaire; A: larve de 10 jours; B : larve de 12 jours; $C$ et $\mathrm{D}$ : régions antérieure et postérieure d'une larve de 13 jours. 

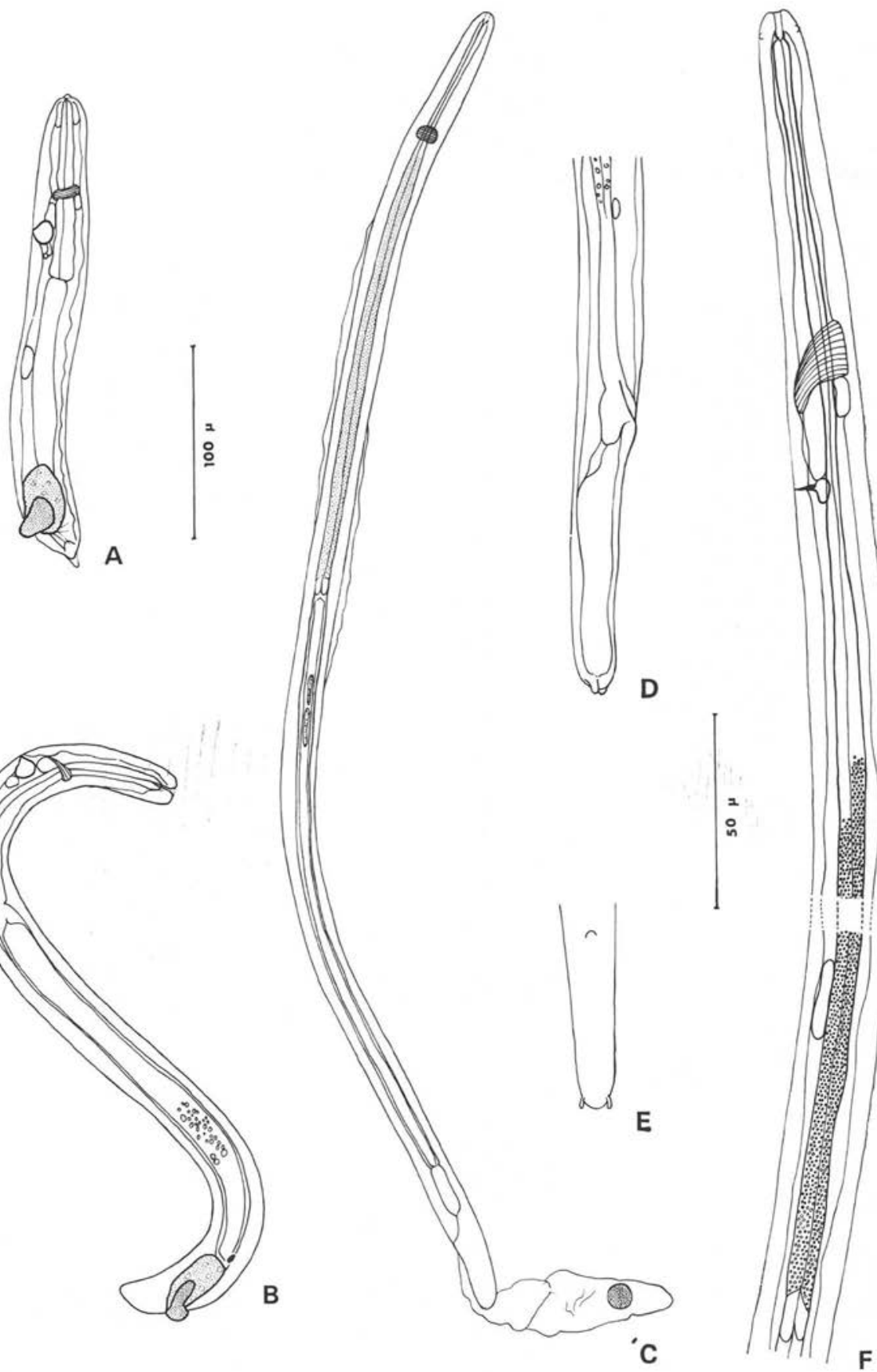

FIG. 4. - Oswaldofilaria petersi ; A, B et $\mathrm{C}$ : aspect général des larves, respectivement à la mue 1 , au stade II et à la mue $2 ; \mathrm{D}$ à $\mathrm{E}$ : stade infestant; $\mathrm{D}$ : région postérieure, vue latérale; E: queue vue ventrale; F: région antérieure d'un stade infestant $q$, vue latérale.

(A à $\mathrm{C}$, éch. $100 \mu ; \mathrm{E}$ à $\mathrm{F}$, éch. $50 \mu$ ). 

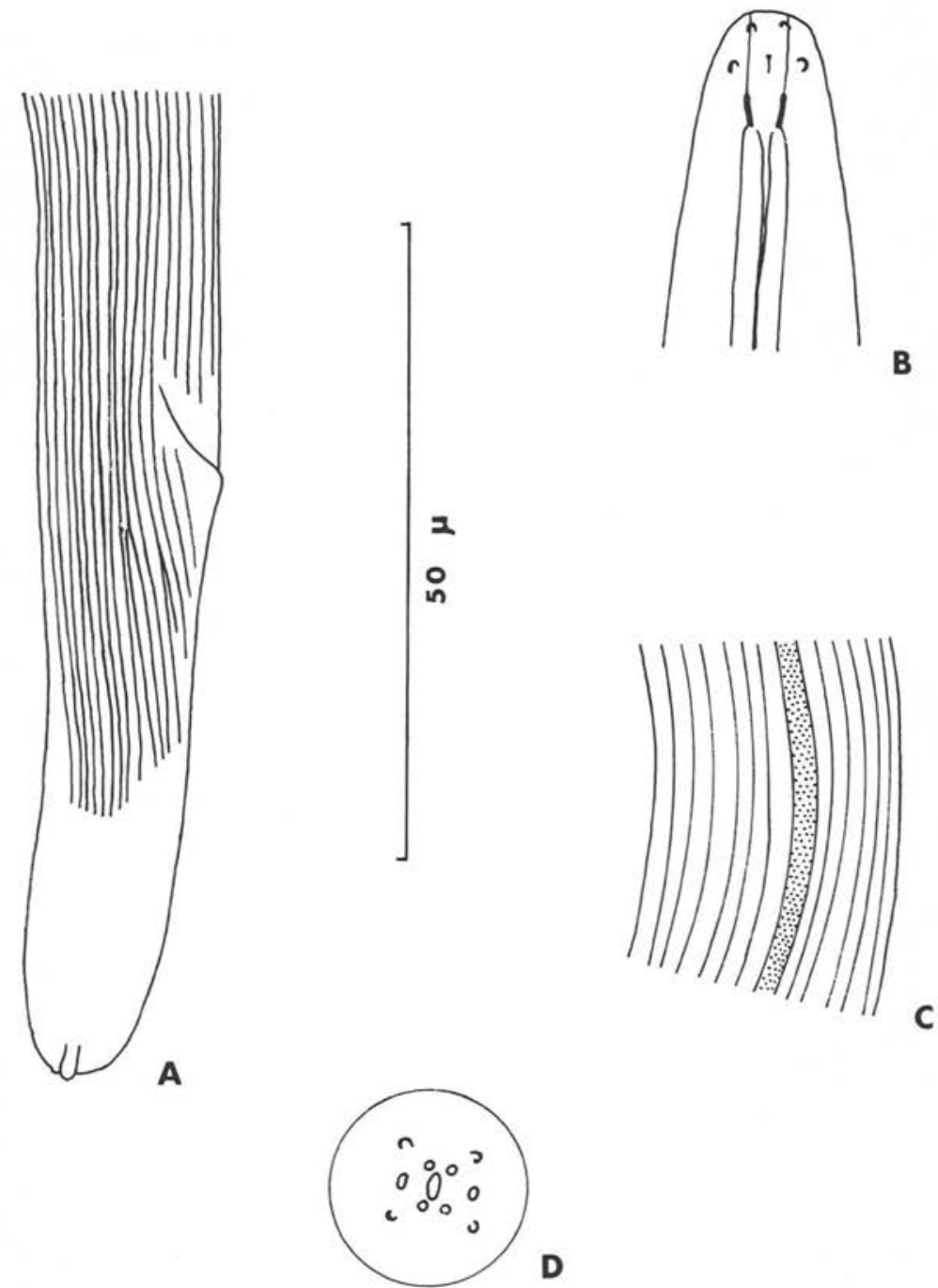

Fig. 5. - Oswaldofilaria petersi; A : ornementation cuticulaire de la région caudale, vue latérale; B: tête, vue latérale; C: ornementation cuticulaire de la région moyenne du corps, vue latérale; D : tête, vue apicale. 
longue, à extrémité arrondie, obtuse, ornée par deux petites languettes latérales subterminales. L'ébauche génitale $\subsetneq$ émigre en avant de la jonction œsophage-intestin mais reste éloignée de la tête (fig. $4 F$ ).

\section{Oswaldofilaria belemensis}

Une cinquantaine de Culex fatigans gorgés sur Dracaena guyanensis sont restés négatifs ; mais 30 Aedes aegypti, gorgés sur le même hôte, puis disséqués 27 jours plus tard, ont permis de recueillir une quinzaine de stades infestants dont quelques spécimens seulement ont été utilisés pour la morphologie, le reste étant gardé pour infester, mais sans succès, un jeune Dracaena.

Microfilaire (fig. $6 \mathrm{~A}$ ) : corps long de $90 \mu$ et large de $8 \mu$; la coloration vitale met seulement en évidence le noyau excréteur à $32 \mu$ de l'apex; le corps interne à $55 \mu$ de l'apex, est formé d'une vacuole contenant un granule très réfringent ; la queue est légèrement en battant de cloche et entièrement nucléée.

Stade infestant (fig. $6 B$ à $F$ ): un stade infestant $q$ récolté dans la trompe du Moustique mesure $870 \mu$ de long et $18 \mu$ de large; la cuticule est ornée d'arêtes longitudinales; anneau nerveux et pore excréteur à $75 \mu$ et $110 \mu$ de l'apex, capsule buccale haute de $8 \mu$; l'œsophage a une portion musculaire longue de $130 \mu$ et une grande portion glandulaire longue de $260 \mu$; queue longue de $52 \mu$ à extrémité légèrement conique ornée par deux languettes latéroventrales subterminales ; l'ébauche génitale est éloignée de $368 \mu$ de l'apex.

\section{Oswaldofilaria spinosa}

Sur une dizaine de Culex fatigans gorgés sur Mabuya microfilarien, un moustique s'est révélé positif avec une larve en mue II située dans l'abdomen (fig. $7 \mathrm{~B}$ ) ; les Culex pipiens s'infestent également mais assez mal ( $2 q$ sur 15 sont positives); deux formes infestantes ont pu être recueillies dans l'abdomen et le thorax, un mois après le gorgement.

Microfilaire (fig. $7 \mathrm{~A}$ ) : corps long de $73 \mu$ et large de $78 \mu$; gaine bien visible aux deux extrémités du corps; tête ogivale ornée de deux pièces céphaliques; la coloration met seulement en évidence le noyau excréteur, à $29 \mu$ de l'apex ; le corps interne est petit et très réfringent ; la queue est nettement atténuée et nucléée.

Stade infestant (fig. $7 C, D, E$ ) $\hat{\jmath}$ : corps long de $980 \mu$, large de $20 \mu$; capsule buccale haute de $7 \mu$; anneau nerveux et pore excréteur à $90 \mu$ et $110 \mu$ de l'apex ; œsophages musculaire et glandulaire longs respectivement de 165 et $420 \mu$; rectum long de $38 \mu$; queue longue de $49 \mu$, à extrémité conique obtuse, ornée par deux languettes subterminales. 


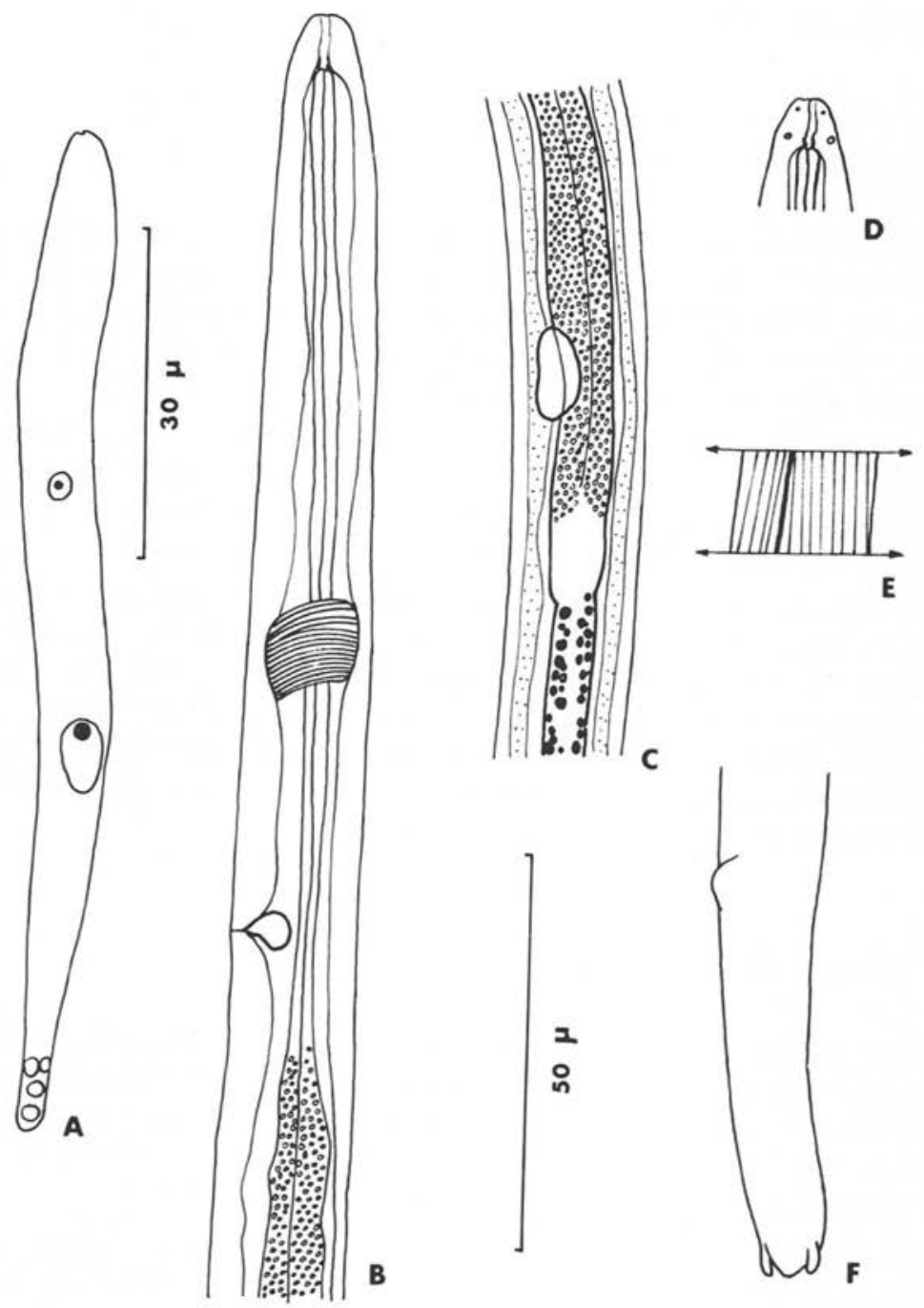

FIG. 6. - Oswaldofilaria belemensis; $\mathrm{A}$ : microfilaire en coloration vitale; $\mathrm{B}$ à $\mathrm{F}$ : stade infestant ; B : région antérieure, vue latérale; $\mathrm{C}$ : région œesophagienne et position de
l'ébauche génitale $\$ ; \mathrm{D}:$ tête en vue médiane; $\mathrm{E}$ : ornementation cuticulare de moyenne du corps, vue latérale; F: queue, vue semi-latérale de la région (A, éch. $30 \mu ; \mathrm{B}$ à $\mathrm{F}$, éch. 50 , vue semi-latérale. 

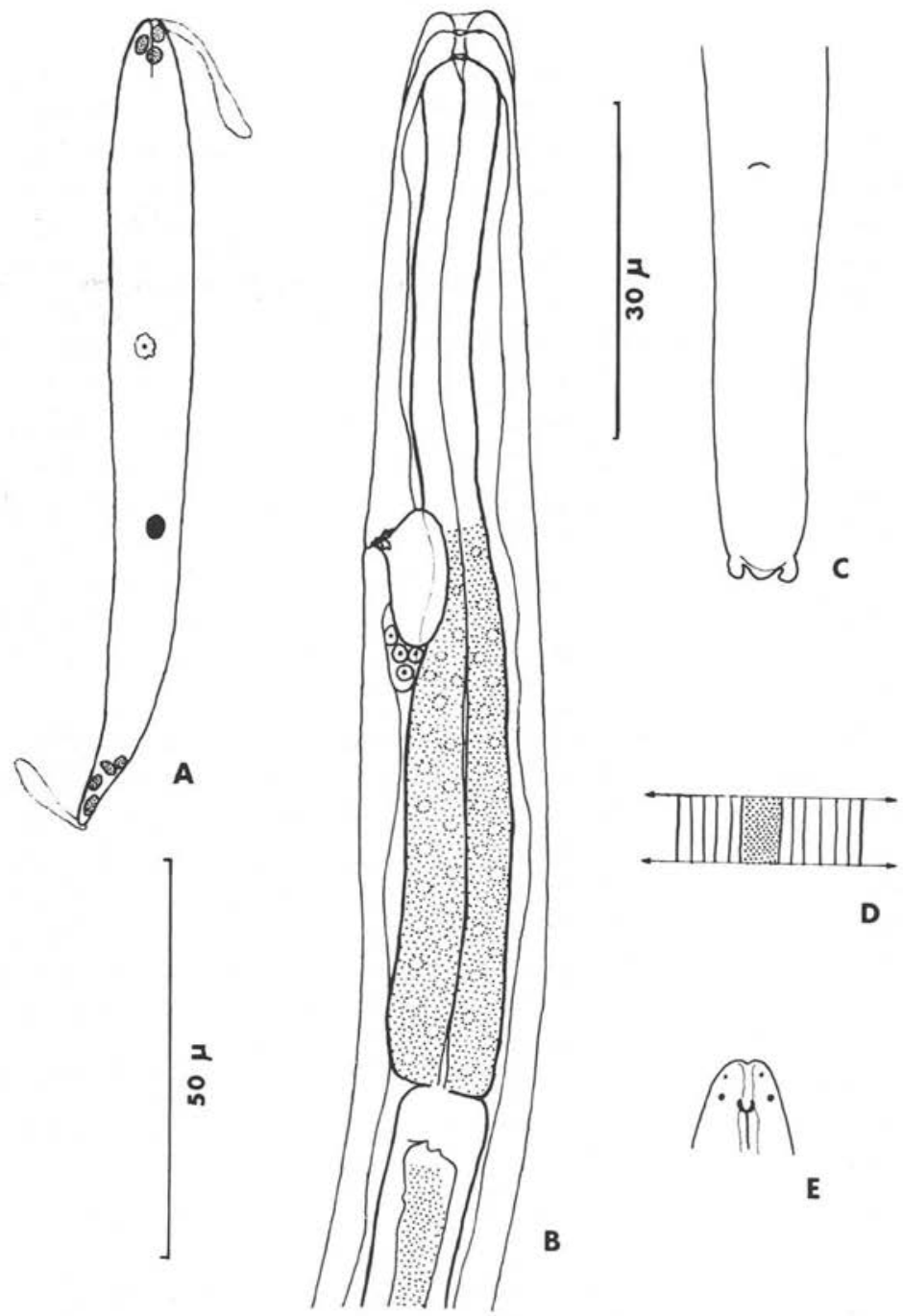

FIg. 7. - Oswaldofilaria spinosa; A : microfilaire en coloration vitale; B : stade II, région antérieure; $\mathrm{C}$ : queue du stade infestant en vue ventrale; $\mathrm{D}$ : ornementation cuticulaire dans la région moyenne du corps; $\mathrm{E}$ : tête, vue médiane.

(A. éch, $30 \mu ; \mathrm{B}$ à E, éch. $50 \mu$ ). 


\section{Conclusion}

Les particularités du développement larvaire qui avaient été observées sur lo filaire d'un Caïman sud-américain, O. bacillaris (Molin, 1858) (cf. Prod'hon et Bain, 1972), se retrouvent chez ces trois Oswaldofilaria, également néotropicaux. Chaque fois que les observations ont pu être faites, les larves sont situées dans le tissu adipeux des moustiques; elles se développent en quinze jours au minimum, la cellule $R_{1}$ se divise assez tardivement (72-96 h), le corps interne persiste plusieurs jours ; enfin le stade infestant, long en moyenne d'un $\mathrm{mm}\left({ }^{*}\right)$, a une cuticule ornée d'arêtes longitudinales, une queue longue $(50 \mu$ environ) munie de deux petites languettes obtuses latérales et subterminales et un œsophage glandulaire bien développé.

Les cycles de deux Oswaldofilaria australiens par Johnston et Mawson (1943) et par Mackerras (1953 et 1962) et celui de Conspiculum guidiensis, en Inde par Pandit, Pandit et Iyer (1930) montrent que, chez toutes ces espèces, le développement s'effectue dans le tissus adipeux des moustiques. Il pourrait ne pas en être ainsi pour les autres Oswaldofilariinae; leur biologie larvaire est encore inconnue et ce fait, qui contraste avec les six cycles élucidés chez le seul genre Oswaldofilaria, semble indiquer que les vecteurs appartiennent à des groupes moins usuels dans les laboratoires que les Moustiques.

Une autre remarque peut être tirée de l'étude de ces cycles; elle concerne la position de l'ébauche génitale chez le stade infestant femelle. Chez les stades infestants des Filaires vivipares, l'ébauche génitale est située à la hauteur de l'intestin chez les larves $\hat{o}$ et plus près de la tête, au niveau de l'œsophage, chez les larves $ᄋ$. Ce dimorphisme, initialement observé chez Dipetalonema viteae (Krepkogorskaya, 1933) a été retrouvé régulièrement. Toutefois, la migration de l'ébauche génitale 우 vers la région antérieure semble nettement moins accentuée chez les Oswaldofilariinae que chez les autres Filaires vivipares, comme le montre la liste des rapports longueur du corps du stade infestant

distance ébauche génitale of-tête pour les 15 espèzes dont la position des ébauches est précisée (Chabaud 1954, Bain, 1971 et 1974 ; Bain et Durette-Desset, 1973, Orihel, 1967).

Oswaldofilariinae : 2,$9 ; 2,3$ et 2,4 respectivement chez $O$. bacillaris, $O$. petersi et O. belemensis.

Dirofilariinae: 4,$5 ; 4,7$ et 4 respectivement chez Foleyella furcata (Linstow, 1899), F. candezei (Fraipont, 1882) et Dirofilaria repens Railliet et Henry, 1911.

Setariinae: 11 chez S. labiatopapillosa (Alessandrini, 1938).

Onchocercinae : 4,25 chez Onchocerca volvulus Leuckart, $1893 ; 7,69$ chez Skrjabinofilaria skrjabini Travassos, $1925 ; 6,84 ; 7,34$ et 5,66 respectivement chez $D$. viteae, $D$.

${ }^{(*)}$ La longueur du stade infestant initialement indiquée pour $O$. bacillaris est de 1600 u ; une nouvelle série d'expériences indique que cette dimension est exceptionnelle, la longueur des stades infestants oscillant entre 950 et $1300 u$. 
dracunculoides (Cobbold, 1870) et D. dessetae Bain, 1973; 3,80 chez Wuchereria bancrofti (Cobbold, 1877) ; 4 chez Brugia tupaiae Orihel, 1966.

Lemdaninae: 2,14 chez Saurositus agamae hamoni (Bain, 1969).

Ces quelques chiffres montrent que chez les Oswaldofilariinae (et les Lemdaninae), le rapport reste proche de 2 ; il est égal ou supérieur à 4 pour les autres sousfamilles et atteint une valeur exceptionnellement élevée (11) pour les Setariinae.

Ces premières données, bien qu'insuffisantes pour attribuer sûrement une valeur phylétique à la position de l'ébauche génitale $\subsetneq$ chez le stade infestant, nous paraissent souligner l'originalité des Oswaldofilariinae.

Elles rendent peu probable, pour cette sous-famille, l'hypothèse d'Anderson (1957) selon laquelle toutes les Filaires vivipares dériveraient de formes sous-cutanées à vulve très antérieure ; cette hypothèse semble par contre pouvoir être envisagée pour d'autres sous-familles et plus particulièrement pour les Setariinae.

\section{Bibliographie}

Anderson (R. C.), 1957. - The life cycles of Dipetalonematid Nematods (Filarioidea, Dipetalonematidae): the problem of their evolution. J. Helm, 31, 203-224.

BaIN (O.), 1972. - Recherches sur la morphogénèse des Filaires chez l'hôte intermédiaire. Ann. Parasit. hum. comp., 47, 251-303.

Bain (O.), 1974. - Développement larvaire de Dipetalonema dessetae, Filaire de Rongeur entretenue au laboratoire. Ann. Parasit. hum. comp., 49, 457-466.

Bain (O.) et Durette-Desset (M.-C.), 1973. - Cycle de Skrjabinofilaria skrjabini, Filaire de Marsupial sud-américain; position systématique. Ann. Parasit. hum. comp., 48, 61-79.

Bain (O.) et Sulahian (A.), 1975. - Trois nouvelles Filaires du genre Oswaldofilaria chez les lézards sud-américains; essai de classification des Oswaldofilariinae. Bull. Mus., $\mathrm{n}^{\circ}$ 232, Zool., 156, 827-841.

Chabaud (A.-G.), 1954. - Sur le cycle évolutif des Spirurides et des Nématodes ayant une biologie comparable. Valeur systématique des caractères biologiques. Ann. Parasit. hum. comp. 29, 42-88, 205-249, 358-425.

Johnston (T. H.) et Mawson (P. M.), 1943. - Remarks on some Nematodes from Australian Reptiles. Trans. Roy. Soc. South Austr., 67, 183-186.

MACKerRas (M.I.), 1953. - Lizard filaria: transmission by mosquitoes of Oswaldofilaria chlamydosauri (Breinl) (Nematoda: Filarioidea). Parasit., 43, 1-3.

Mackerras (M. I.), 1962. - Filarial parasites (Nematoda: Filarioidea) of Australian animals. Aust. J. Zool., 10, 400-457.

ORIHEL (T. C.), 1967. - Development of Brugia tupaiae in the intermediate and definitive hosts. J. Parasit.. 53, 376-381.

Pandit (C. G.), Pandit (S. R.) et Iyer (P. V. S.), 1930. - The development of the Filaria Conispiculum guidiensis (1929) in C. fatigans, with a note on the transmission of the infection. Ind. J. Med. Res., 17, 421-429.

Prod'hon (J.) et Bain (O.), 1972. - Développement larvaire chez Anopheles stephensi d'Oswaldofilaria bacillaris, Filaire de Caïman sud-américain, et redescription des adultes. Ann. Parasit. hum. comp., 47, 745-758. 